\title{
Mapping low-latitude stellar substructure with SEGUE photometry
}

\author{
Jelte T. A. de Jong ${ }^{1}$, Brian Yanny ${ }^{2}$, Hans-Walter Rix ${ }^{1}$, Eric F. Bell ${ }^{1}$ \\ and Andrew E. Dolphin ${ }^{3}$ \\ ${ }^{1}$ Max-Planck-Institut für Astronomie, Königstuhl 17, 69117 Heidelberg, Germany \\ email: dejong@mpia.de \\ ${ }^{2}$ Fermi National Accelerator Laboratory, P.O. Box 500, Batavia, IL 60510, United States \\ ${ }^{3}$ Raytheon Corporation, 870 Winter Street, Waltham, MA 02451, United States
}

\begin{abstract}
Encircling the Milky Way at low latitudes, the Low Latitude Stream is a large stellar structure, the origin of which is as yet unknown. As part of the SEGUE survey, several photometric scans have been obtained that cross the Galactic plane, spread over a longitude range of $50^{\circ}$ to $203^{\circ}$. These data allow a systematic study of the structure of the Galaxy at low latitudes, where the Low Latitude Stream resides. We apply colour-magnitude diagram fitting techniques to map the stellar (sub)structure in these regions, enabling the detection of overdensities with respect to smooth models. These detections can be used to distinguish between different models of the Low Latitude Stream, and help to shed light on the nature of the system.
\end{abstract}

Keywords. Galaxy: stellar content - Galaxy: structure

\section{Introduction}

During the past decade, the structure of the Milky Way (MW) has been mapped in unprecedented detail, owing to deep, wide-field photometric surveys, such as the Sloan Digital Sky Survey (SDSS, York et al. 2000) and 2MASS. Apart from an improved understanding of the overall shape and stellar populations of the MW, these data have unveiled a plethora of substructures on all scales. Two examples of large structures are the stellar overdensity towards Canis Major (CMa, Martin et al. 2004), and the Low Latitude Stream (LLS), or Monoceros stream, a ring-like structure that seems to encircle the MW at low latitudes (Newberg et al. 2002, Ibata et al. 2003).

The origin of these structures is as yet unclear, but several hypotheses have been put forward. CMa might be (the remnant of) an accreted dwarf galaxy (e.g. Martin et al. 2004, Martínez-Delgado et al. 2005, Bellazzini et al. 2006, Butler et al. 2007, de Jong et al. 2007) with the LLS being tidal debris stripped from CMa. Martin et al. (2005) and Peñarrubia et al. (2005) have shown that dynamical models of such an accretion can indeed reproduce both the CMa overdensity as well as the LLS. On the other hand, as these structures are located at very low Galactic latitudes, it cannot be ruled out that they are intrinsic to the disk itself (e.g. Momany et al. 2006). Another explanation for the LLS is that its stars originate in the disk, but have been pulled out of the plane by interactions with satellites (e.g. Kazantzidis et al. 2007, Younger et al. 2008).

Here we present preliminary results of the application of colour-magnitude diagram (CMD) fitting techniques to photometry at low Galactic latitudes taken as part of the SEGUE survey. These techniques, developed in de Jong et al. (2008), allow us to map the 3-D distribution of stars. Fig. 1 demonstrates that the resulting maps of stellar 

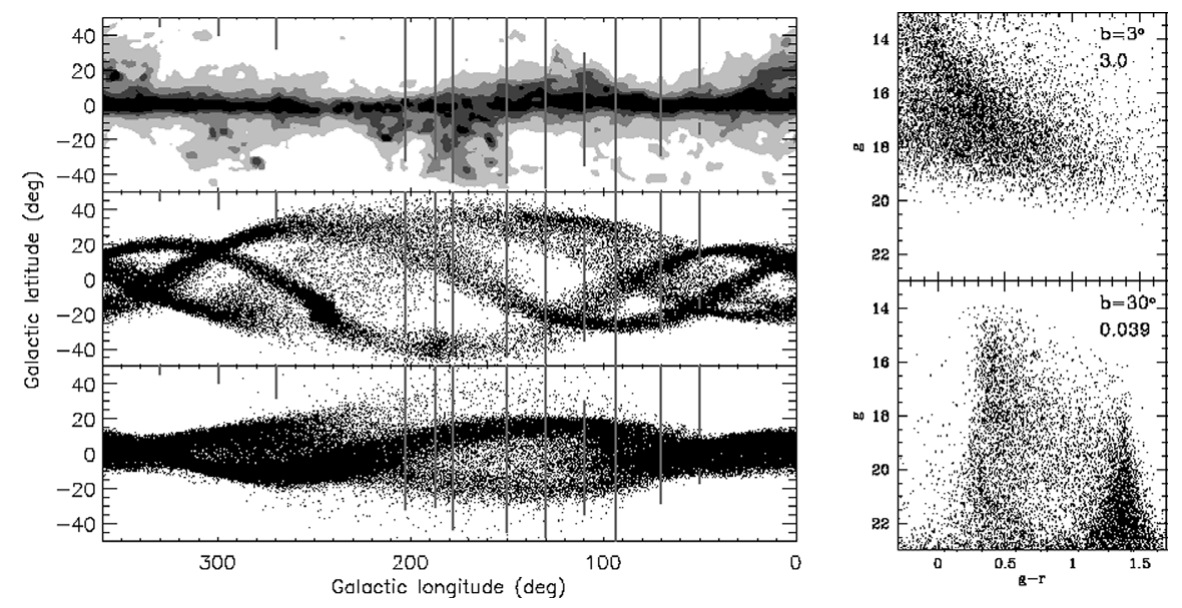

Figure 1. Left: Overview of imaging scans used for this analysis, indicated as vertical lines, in Galactic coordinates. The top panel shows reddening according to the dust extinction maps from Schlegel et al. (1998), with the gray scale indicating regions with $E(B-V)>0.1,0.25$, 0.5 and 1.0 mag. The middle and lower panels show the LLS models of Peñarrubia et al. (2005) and Martin et al. (2005), respectively. Right: CMDs from the scan at $l=94^{\circ}$. The top CMD, at $b=3^{\circ}$, is heavily extincted (E(B-V) $\left.=3 \mathrm{mag}\right)$, while the bottom CMD, at $b=30^{\circ}$, is typical of the data used for our CMD fitting analysis.

(sub)structure along the SEGUE imaging scans can help to distinguish between different models of the LLS and provide crucial constraints for further modelling endeavours.

\section{Data and methods}

SEGUE (Sloan Extension for Galactic Understanding and Exploration), an imaging and spectroscopic survey aimed at the study of the MW and its stellar populations, is one of the constituent projects of the extended SDSS survey (SDSS II). The photometric part of the SEGUE survey consists of several $2.5^{\circ}$ wide scans going through the Galactic plane (see Fig. 1), allowing a view of the Galaxy at low latitudes. For the CMD-fitting analysis we restrict ourselves to the two most sensitive bands, $g$ and $r$, which are complete to $\sim 22$ nd magnitude. Although for the main survey the photometric accuracy is at least $2 \%$ down to these limits (Ivezic et al. 2004), in crowded regions at low latitudes the accuracy might be worse and the calibration in the data used here is preliminary. In Fig. 1 the coverage of the scans between Galactic latitudes of $+50^{\circ}$ and $-50^{\circ}$ is shown. Where possible the SEGUE scans were extended to high latitudes by extracting $2.5^{\circ}$ wide strips from SDSS data release 5 (Adelman-McCarthy et al. 2007). We de-redden all data using the dust maps from (Schlegel et al. 1998), including the correction suggested by Bonifacio et al. (2000). Two CMDs from the scan at $l=94^{\circ}$ are also shown in Fig. 1 . Very close to the plane, the reddening is very high and de-reddening is unable to correct this accurately. As poor de-reddening would limit the accuracy of our results, we avoid regions with reddening higher than $\mathrm{E}(\mathrm{B}-\mathrm{V})=0.2 \mathrm{mag}$. The $\mathrm{CMD}$ in the bottom right of Fig. 1 is representative of the the data used.

In CMD fitting, observed photometry is compared with models in order to constrain the constituent stellar populations of stellar systems. Traditionally, CMD fitting has been used mostly to determine star formation histories and age-metallicity relations of isolated objects, such as dwarf galaxies and globular clusters. We use the CMD fitting 


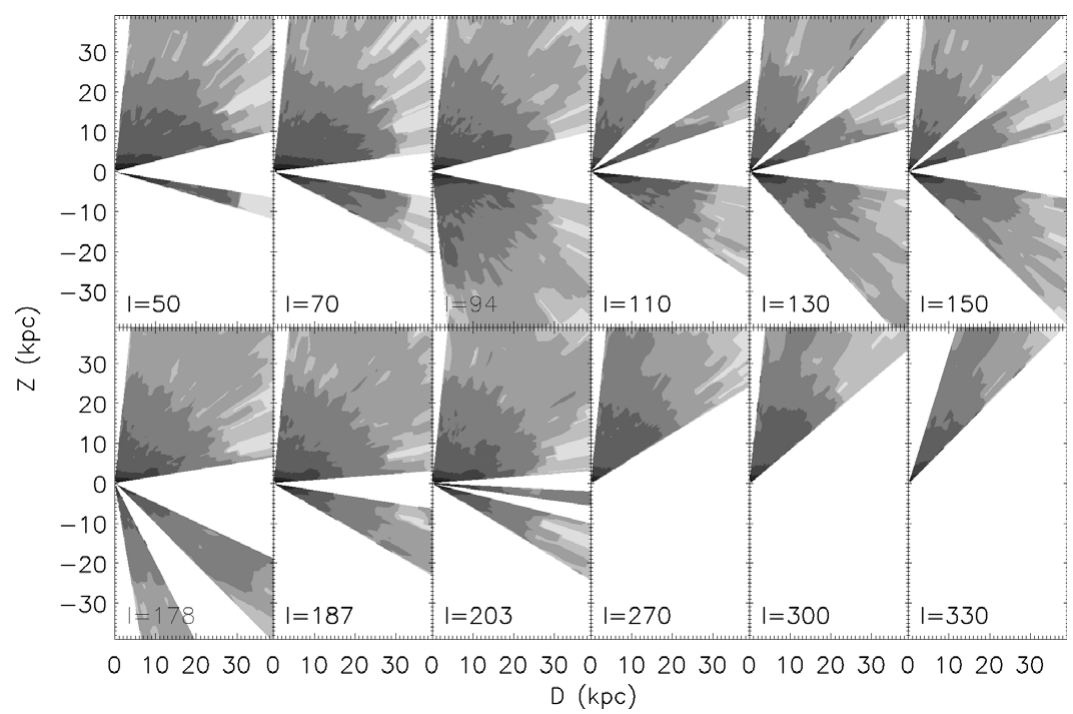

Figure 2. Stellar mass density resulting from the single population fits, as function of distance from the sun along the Galactic plane, and height above or below the plane, in kpc. Each panel shows a different imaging scan, the Galactic longitude of which is listed at the bottom, and darker gray levels correspond to higher densities.

software package MATCH (Dolphin 2001), adapted to solve for distance rather than agemetallicity evolution, to fit the distance distribution of stars along the line-of-sight. A demonstration and a detailed description of this technique and its application to SDSS data can be found in de Jong et al. (2008). Here we shortly discuss the basic approach used in the current analysis.

Observed CMDs are fit with a set of model CMDs, each of which corresponds to a certain age, metallicity and distance. The models are created by populating theoretical isochrones from Girardi et al. (2004) and convolving them with the SDSS photometric errors and completeness (see de Jong et al. 2008). Using maximum-likelihood methods, MATCH determines the best-fitting linear combination of model CMDs, thereby providing the distribution of stars over age-metallicity-distance space. We do this in two ways. First, fitting a narrow colour range of $0.3<g-r<0.8$ and a single combination of age and metallicity $([\mathrm{Fe} / \mathrm{H}]=-0.8,8 \mathrm{Gyr})$; this corresponds to measuring the density of main-sequence stars as function of distance modulus. Second, we use a wider colour range $(0.1<g-r<0.8)$ that includes turn-off stars, providing information on age and metallicity, and fit for three different stellar populations: a 'thick-disk' population with $[\mathrm{Fe} / \mathrm{H}]=\sim-0.8$ and $t=10 \mathrm{Gyr}$, a 'halo' population with $[\mathrm{Fe} / \mathrm{H}] \sim-1.3$ and $t=13 \mathrm{Gyr}$, and a broad 'general' population with $[\mathrm{Fe} / \mathrm{H}]=\sim-0.8$ and $5<t<14 \mathrm{Gyr}$.

\section{Results}

From the fits described above we obtain the stellar mass in bins of constant distance modulus. This can be converted to spatial stellar mass density, giving contour maps such as the ones presented in Fig. 2 for the single population fits. As expected, the density is clearly seen to decrease with increasing distance from the plane and from the Galactic center. Fig. 3 is colour-coded with the type of population, based on the results from the fits with the thick-disk-like (red), halo-like (blue) and very broad (green) populations. It is clear that the thick disk and halo are indeed fit with the appropriate populations, 


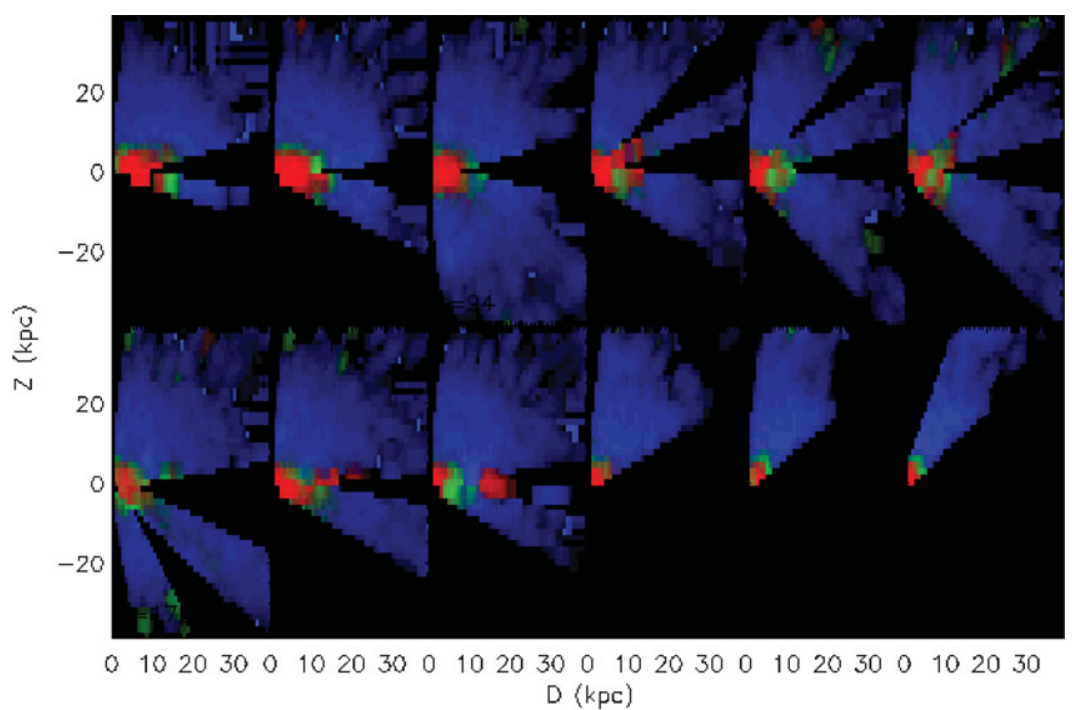

Figure 3. Distribution of stellar populations, following the fit results for three different populations. Each panel is for a different longitude, following the same lay-out as Fig. 2. The thick-disk-like population with $[\mathrm{Fe} / \mathrm{H}]=\sim-0.8$ and $t=10 \mathrm{Gyr}$ is colour-coded as red, the halolike population with $[\mathrm{Fe} / \mathrm{H}] \sim-1.3$ and $t=13 \mathrm{Gyr}$ as blue, and the broad population with $[\mathrm{Fe} / \mathrm{H}]=\sim-0.8$ and $5<t<14$ Gyr as green.

with the third, broad population mostly visible in the regions where the halo and disk populations have similar densities.

To increase the contrast of any substructures on top of the 'smooth' distribution of stars, a smooth model must be subtracted from the stellar density maps. We assume a model with a double exponential thin and thick disk and an axisymmetric power-law halo. Since the constraints on the disk are limited due to a lack of data at the smallest distances and the masking out of the lowest latitudes, we fix the thin and thick disk scale lengths at 2.6 and $3.6 \mathrm{kpc}$, respectively, and their scale heights at 0.3 and $1.0 \mathrm{kpc}$, respectively. Using $R_{\odot}=7.6 \mathrm{kpc}$ (following Vallée (2008)), our best-fit model gives a local thin disk density of $0.08 M_{\odot} \mathrm{pc}^{-3}$, local thick disk and halo normalisations of 0.058 and 0.0016 , and an almost round $(q=0.9)$ halo with a power-law index of -3 . These fit values agree well with previous determinations (see e.g. Siegel et al. 2002), although our fit favours a rounder halo than found in previous SDSS studies by Bell et al. (2008) and Juric et al. (2008). For the fits with three populations the model favours a lower thin disk density and higher normalisations for the thick disk and halo. This is due to the lack of a specific thin disk-like population in the fits. The residuals left after subtracting the best-fitting smooth models from the stellar density maps are shown in Fig. 4. In this figure we have zoomed in to regions close to the plane, where the LLS is expected to be present.

\section{Discussion and Conclusions}

Colour-magnitude diagram fitting can successfully reproduce the distance distribution of stars. This is corroborated by the fact that the disk and halo model that best fits the density distributions in Fig. 2 gives densities and density ratios between the different model components that are in good agreement with previous determinations from star counts (e.g. Siegel et al. 2002 and references therein). 

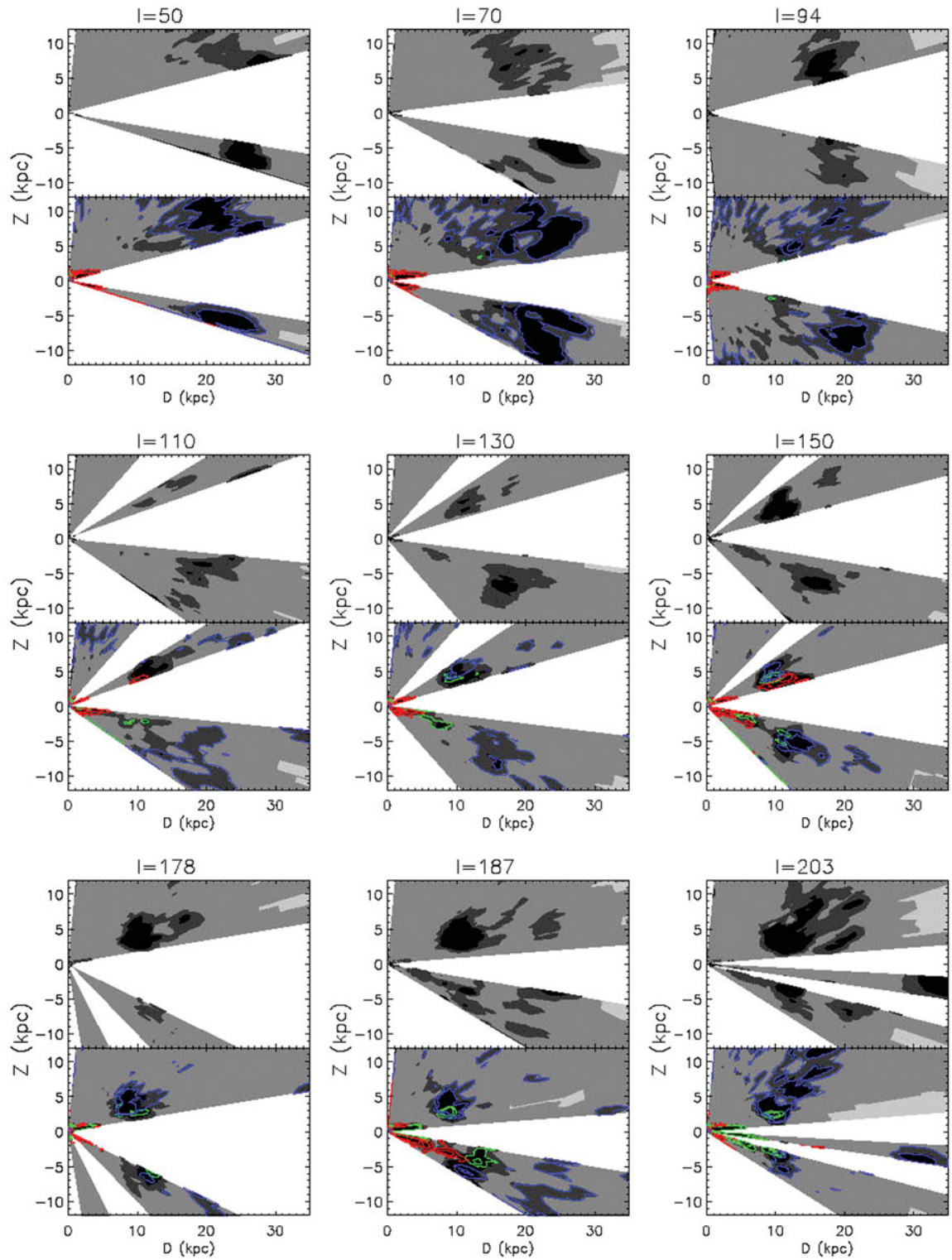

Figure 4. Overdensities at low Galactic latitudes. Residual maps for both single population (upper subpanels) and triple population (lower subpanels) fits for all stripes that cross the Galactic plane. Starting from black, the gray scale levels correspond to areas with residual densities $>2,2>1,1>-1$, and $<-1$ times the model density. White areas contain no information due to an absence of data or too high reddening. Coloured contours show overdensities of $>2,2>1$ times the model density in the individual thick disk-like (red), halo-like (blue) and broad (green) populations.

After subtraction of a smooth Galaxy model, a wealth of substructure becomes visible at low Galactic latitudes (Fig. 4). When searching for pieces of the LLS, possible other overdensities need to be taken into account. The scans at $l=50^{\circ}$ and $70^{\circ}$ cross the Hercules-Aquila cloud (Belokurov et al. 2007), a large overdensity extending both above and below the plane to high latitudes at a distances between 10 and $20 \mathrm{kpc}$. 
Consequently, the extended overdensities seen in these scans in Fig. 4 are likely to be related to this structure (it appears slightly more distant in our results because HerculesAquila is more metal-poor than the populations used in our fits). Furthermore, the Sagittarius stream lies partly along the scan at $l=203^{\circ}$, and is probably partly responsible for the overdensities there. However, even with these restrictions, a large number of overdensities is left, of which at least part corresponds to the LLS. For example, the overdensities at $\mathrm{D}=10 \mathrm{kpc}, \mathrm{Z}=+5 \mathrm{kpc}$ in the $l=178^{\circ}$ and $l=187^{\circ}$ scans correspond to the original detection of the Monoceros overdensity by Newberg et al. (2002).

More accurate analysis of the distances and metallicities of the detected overdensities is needed to piece together an overview of the LLS and possible other substructures. One avenue of solving for the degeneracies between distance, age, and metallicity is by using colour-colour information to obtain metallicity estimates (Ivezic et al. 2008). With locations and metallicities for the overdensities in place, a detailed picture of the LLS will emerge that can help to shed light on the nature of this structure.

Funding for the SDSS and SDSS-II has been provided by the Alfred P. Sloan Foundation, the Participating Institutions, the National Science Foundation, the U.S. Department of Energy, the National Aeronautics and Space Administration, the Japanese Monbukagakusho, the Max Planck Society, and the Higher Education Funding Council for England. The SDSS Web Site is http://www.sdss.org/.

\section{References}

Adelman-McCarthy et al. 2007, ApJS, 172, 634

Bell, E. F., et al. 2007, ApJ, 680, 295

Bellazzini, et al. 2006, MNRAS, 366, 865

Belokurov, V., et al. 2007, ApJ, 657, L89

Bonifacio, P., et al. 2000, AJ, 120, 2065

Butler, D. J. et al. 2007, $A J, 133,2274$

de Jong, J. T. A., et al. 2007, ApJ, 662, 259

de Jong, J. T. A., et al. 2008, AJ, 135, 1361

Dolphin, A. E. 2001, MNRAS, 332, 91

Girardi, L., et al. 2004, Aछ A, 422, 205

Ibata, R. A., et al. 2003, MNRAS, 340, L21

Ivezić, Ž. et al. 2004, $A N, 325,583$

Ivezić, Ž. et al. 2008, ApJ, subm., arXiv:0804.3850

Jurić, M., et al. 2008, ApJ, 673, 864

Kazantzidis, S., et al. 2007, ApJ, subm. (arXiv:0708.1949)

Martin, N. F., et al. 2004, MNRAS, 348, 12

Martin, N. F., et al. 2005, MNRAS, 362, 906

Martínez-Delgado, D., et al. 2005, ApJ, 633, 205

Momany, Y., et al. 2006, A\&A A 451, 515

Newberg, H. J., et al. 2002, ApJ, 569, 245

Peñarrubia, J., et al. 2005, ApJ, 626, 128

Schlegel, D., Finkbeiner, D. \& Davis, M. 1998, ApJ, 500, 525

Siegel, M. H., et al. 2002, ApJ, 578, 151

York, et al. 2000, AJ, 120, 1579

Younger, J. D., et al. 2008, ApJ, 676, L21 